\title{
Are Abell Clusters Correlated with Gamma-Ray Bursts?
}

\author{
K. Hurley \\ University of California, Space Sciences Laboratory, Berkeley, CA 94720-7450 \\ D. Hartmann \\ Clemson University, Dept. of Physics and Astronomy, Clemson SC 29634-1911 \\ C. Kouveliotou, G. Fishman \\ NASA Marshall Space Flight Center, ES-62, Huntsville AL 35812 \\ J. Laros \\ Lunar and Planetary Laboratory, University of Arizona, Tucson, AZ 85721 \\ T. Cline \\ NASA Goddard Space Flight Center, Code 661, Greenbelt, MD 20771 \\ M. Boer \\ CESR, 9, avenue du Colonel Roche, 31029 Toulouse, France
}

Received — 


\begin{abstract}
A recent study has presented marginal statistical evidence that gamma-ray burst sources are correlated with Abell clusters, based on analyses of bursts in the BATSE 3B catalog. Using precise localization information from the 3rd Interplanetary Network, we have reanalyzed this possible correlation. We find that most of the Abell clusters which are in the relatively large 3B error circles are not in the much smaller IPN/BATSE error regions. We believe that this argues strongly against an Abell cluster-gamma-ray burst correlation.
\end{abstract}

Subject headings: galaxies: clusters: general gamma rays: bursts 


\section{Introduction}

A correlation between the positions of gamma-ray bursts (GRBs) determined by the Burst and Transient Source Experiment (BATSE) aboard the Compton Gamma-Ray Observatory and those of rich, nearby Abell clusters was recently claimed by Kolatt and Piran ( 1996) (hereafter KP). They analyzed the BATSE 3B catalog (Meegan et al. 1996) in conjunction with data on Abell clusters (Abell, Corwin, \& Olowin 1989), and concluded that these gamma-ray bursts were correlated with them at the $95 \%$ confidence level. In their study, they selected the $3 \mathrm{~B}$ bursts with error circle radii $\lesssim 2.77^{\circ}$, and bursts and clusters with $|b|>30^{\circ}$. They then calculated the number of burst-cluster pairs, $\mathrm{N}(\theta)$, whose separation was smaller than a given angle $\theta$, for $\theta=1^{\circ}-6^{\circ}$. Comparing this number to the numbers found for randomly generated catalogs, they found a number for $\theta=4^{\circ}$ that was significant at the $95 \%$ confidence level.

If such findings could be confirmed, they would constitute statistical evidence for counterparts to GRB sources, and would indicate that at least some GRBs are at cosmological distances. We have attempted to confirm these results by subjecting them to more stringent tests. The positions of those Abell clusters which appear to be related to GRBs, based on their locations within BATSE error circles, must be consistent with all known localization information for the bursts in question. In particular, they must also lie within the annuli or the error boxes of the 3rd Interplanetary Network (IPN3) for those bursts. Below we present the IPN3 data, the results of our test, and our conclusions.

\section{IPN3 Data}

For the period covered by the 3B catalog, IPN3 consisted of the Ulysses spacecraft, at distances up to 6 AU (Hurley et al. 1992), BATSE, and, until mid-1992, Pioneer Venus 
Orbiter (PVO). When only Ulysses and BATSE observed a burst, the resulting localization was an annulus generally crossing the BATSE error circle. When PVO observed the burst also, an error box resulted with dimensions in the several arcminute range in the best cases. Examples may be found in Hurley et al. (1993) and Cline et al. (1992). The annuli widths varied from about 10" to 1000", with average width 5.7'. Descriptions of the data set have appeared in Hurley et al. ( 1994, 1996). Of the 136 "accurately" localized BATSE bursts selected by KP on the basis of their error circle size and galactic latitude, 93 have IPN3 annuli associated with them, and 10 have IPN3 error boxes. The total area covered by the 10 error boxes, plus the 93 intersections of the IPN3 and BATSE locations, is less than the area covered by the corresponding BATSE error circles alone, by at least an order of

magnitude. This substantially reduces the probability of chance correlations between Abell clusters and GRB positions, and results in a stronger test.

\section{Results of the Test}

The all-sky catalog of Abell clusters (Abell, Corwin, \& Olowin 1989) contains 4073 rich clusters, each having at least 30 bright members, and covers redshifts less than $\mathrm{z}=0.2$. Following KP, a latitude cut of $|\mathrm{b}| \geq 30^{\circ}$ was applied to both cluster and burst catalogs. We began by verifying the numbers of bursts and Abell clusters in the KP study, 136 (after latitude and error circle size cuts are applied) and 3616 (after the latitude cut), respectively. For each cluster, we then checked each of the BATSE error circles to see whether their positions were consistent. If it was, we finally checked for consistency with any IPN3 location information. Since, in the KP study, Abell clusters within $4^{\circ}$ of a BATSE position were considered to be correlated with the burst, we assigned an error radius of $4^{\circ}$ to the BATSE bursts, and also used this to define the BATSE/IPN3 error box. An example is shown in figure 1. 
We found that 1260 Abell clusters lie in the BATSE $4^{\circ}$ radius error circles. These circles cover about $16.6 \%$ of the sky, and given the density of Abell clusters in the sample, about 1200 would be expected to fall in the error circles by chance. This is consistent with the KP result in that it represents about a $2 \sigma$ excess of Abell clusters in these error circles, assuming a negligible cluster- cluster correlation. The BATSE/IPN error boxes cover $3.3 \times 10^{5}$ square arcminutes, or $1.9 \%$ of the total area of the BATSE error circles that they intersect. We found that 14 Abell clusters lie within the BATSE/IPN3 error boxes, while 16 are expected by chance. This indicates that the overwhelming majority of the Abell clusters which are in BATSE error circles are there by chance.

We reanalyzed the data using the standard $1 \sigma$ BATSE error circle radii, defined as $\left(\sigma_{\text {sys }}^{2}+\sigma_{\text {stat }}^{2}\right)^{1 / 2}$, where $\sigma_{\text {sys }}$ is the systematic error, $1.6^{\circ}$, and $\sigma_{\text {stat }}$ is the statistical error, given in the BATSE 3B catalog for each burst. This results in smaller error circles, covering $3.5 \%$ of the sky. The BATSE/IPN3 error boxes cover $4.5 \%$ of the total area of the BATSE error circles that they intersect, or $1.2 \times 10^{5}$ square arcminutes, and 5.7 Abell clusters should fall in them by chance. 4 were found to actually lie in them.

\section{Discussion}

If gamma-ray bursts indeed originate at cosmological distances, their observed brightness distribution suggests that BATSE samples bursts to a redshift of order unity (e.g., Wickramasinghe et al. 1993). To yield the all-sky rate of $\sim 10^{3} \mathrm{yr}^{-1}$ an average galaxy must produce one observable burst every $10^{6} \mathrm{yrs}$, corresponding to a mean comoving galaxy density of $n_{0}=10^{-2} \mathrm{Mpc}^{-3}$. In the local part of the universe approximate distances are given by $\mathrm{D}=\mathrm{z} \mathrm{L}_{\mathrm{H}}$, where the Hubble distance is defined as $\mathrm{L}_{\mathrm{H}}=\mathrm{c} / \mathrm{H}_{0} \sim 3,000 \mathrm{~h}^{-1} \mathrm{Mpc}$, where $\mathrm{h}$ is the Hubble constant in units of $100 \mathrm{~km} \mathrm{~s}^{-1} \mathrm{Mpc}^{-1}$. The depth of the Abell cluster sample is thus about $600 \mathrm{~h}^{-1} \mathrm{Mpc}$. 
Only $\sim 2 \%$ of all observable GRBs are thus expected to occur within the volume of space sampled by the Abell catalog. Even if we assume that all galaxies are correlated with clusters, to obtain a significant correlation of bursts with clusters would thus require that either the BATSE sampling redshift is much less than unity, and/or that all well-localized bursts are approximately confined to the sampling volume of the cluster catalog. KP obtain a sample of 549 bursts after applying their first cut (in latitude). We expect $\sim 2 \%$ of these (11) to be correlated with clusters. As shown above, concidences are expected in 5.7 cases, and the observed number of coincidences is 4 .

The KP results indicate a sampling distance (for all bursts) of $\mathrm{z}_{\max }=0.7$ and a sampling distance of $\mathrm{z}_{\mathrm{a}}=0.31$ for the 136 "accurately" localized bursts, which implies that $35( \pm 20)$ bursts are within the Abell range. Thus, in addition to the number of chance coincidences between Abell clusters and the IPN3 positions (5.7), one expects 15-55 more, which is clearly in contradiction with our findings.

We believe that the Abell cluster-gamma-ray burst correlation found by KP is best explained by statistics. There is a $5 \%$ chance of finding a correlation with any set of cataloged objects in their study, and certainly many catalogs have been searched for GRB counterparts. Indeed, as soon as precise GRB positions began to become available from the 1st IPN, they were subjected to extensive catalog searches, including Abell clusters, (e.g. Hurley 1982, Barat et al. 1984) and it seems unlikely that an Abell cluster correlation would have been missed.

If a correlation were to exist between Abell clusters and GRBs, the poor angular resolution of BATSE, the small sample size for the IPN3 positions, and the small number of low redshift events reduce our chances of detecting it. However, Abell clusters are somewhat concentrated towards the super-galactic plane to distances perhaps as large as $\sim 300 \mathrm{~h}^{-1}$ Mpc (e.g., Tully 1987 ; but see Postman et al. 1989 ) and one might thus expect to find a 
significant global anisotropy of bright bursts tracing the galaxies within $\mathrm{z} \sim 0.1$. This effect could be noticeable even if only poor localizations were available. So far, the search for a super-galactic anisotropy has not been successful (Hartmann et al. 1996).

While attempts to find GRB counterparts through correlation analysis with galaxies or clusters of galaxies may provide some evidence for a cosmological burst origin, we caution that the present data are insufficient to prove that connection. Five more years of BATSE/IPN3 data may be sufficient for this task. Another solution would be to improve burst localization accuracy to the arcsecond regime, so that one would not have to rely on statistics, but simply on the inspection of such error boxes. This may become possible with ongoing searches for optical transients, through future imaging burst detectors, or an improved triangulation network. All of these possibilities are being pursued actively at the present time.

KH is grateful for Ulysses support under JPL Contract 958056, and IPN3 support under NASA NAG 5-1560. DH acknowledges support from NASA through the COMPTON Observatory guest investigator program. We also acknowledge the helpful comments of Chip Meegan, and the careful reading and constructive criticism of T. Kolatt and T. Piran. 


\section{REFERENCES}

Abell, G. O., Corwin, H. G., \& Olowin, R. P. 1989, ApJS, 70, 1

Barat, C., et al. 1985, ApJ, 280, 150

Cline, T. et al. 1992 in Gamma-Ray Bursts, ed. W. Paciesas \& G. Fishman (New York: AIP), 72

Hartmann, D. H., Briggs, M. S., \& Mannheim, K. 1996, in Gamma-Ray Bursts - Third workshop, ed. M. Briggs, C. Meegan, and C. Kouveliotou, (New York: AIP), in press

Hurley, K. 1982 in Gamma-Ray Transients and Related Astrophysical Phenomena, ed. R. Lingenfelter, H. Hudson, and D. Worrall (New York: AIP), 85

Hurley, K. et al. 1992 Astron. Astrophys. Suppl. Ser. 92(2) 401

Hurley, K. et al. 1993 Astron. Astrophys. Suppl. Ser. 97(1) 39

Hurley, K. et al. 1994 in Gamma-Ray Bursts - Second Workshop, ed. G. Fishman, J. Brainerd, \& K. Hurley (New York: AIP), 27

Hurley, K. et al. 1996 in 3rd Huntsville Symposium on Gamma-Ray Bursts, ed. C. Kouveliotou, M. Briggs, and G. Fishman (New York: AIP), in press

Kolatt, T., and Piran, T. 1996, ApJ, 467, L41

Meegan, C. et al. 1996 ApJS, 106, 45

Postman, M., Spergel, D. N., Sutin, B., \& Juszkiewicz, R. 1989, ApJ, 346, 588

Tully, R. B. 1987 ApJ, 323, 1

Wickramasinghe, W. A. D. T. et al. 1993, ApJ, 411, L55 
Fig. 1.- The field around BATSE burst 121. The BATSE $4{ }^{\circ}$ radius error circle and the Ulysses/BATSE triangulation annulus are shown. None of the Abell clusters in this field lies within the triangulation annulus. 


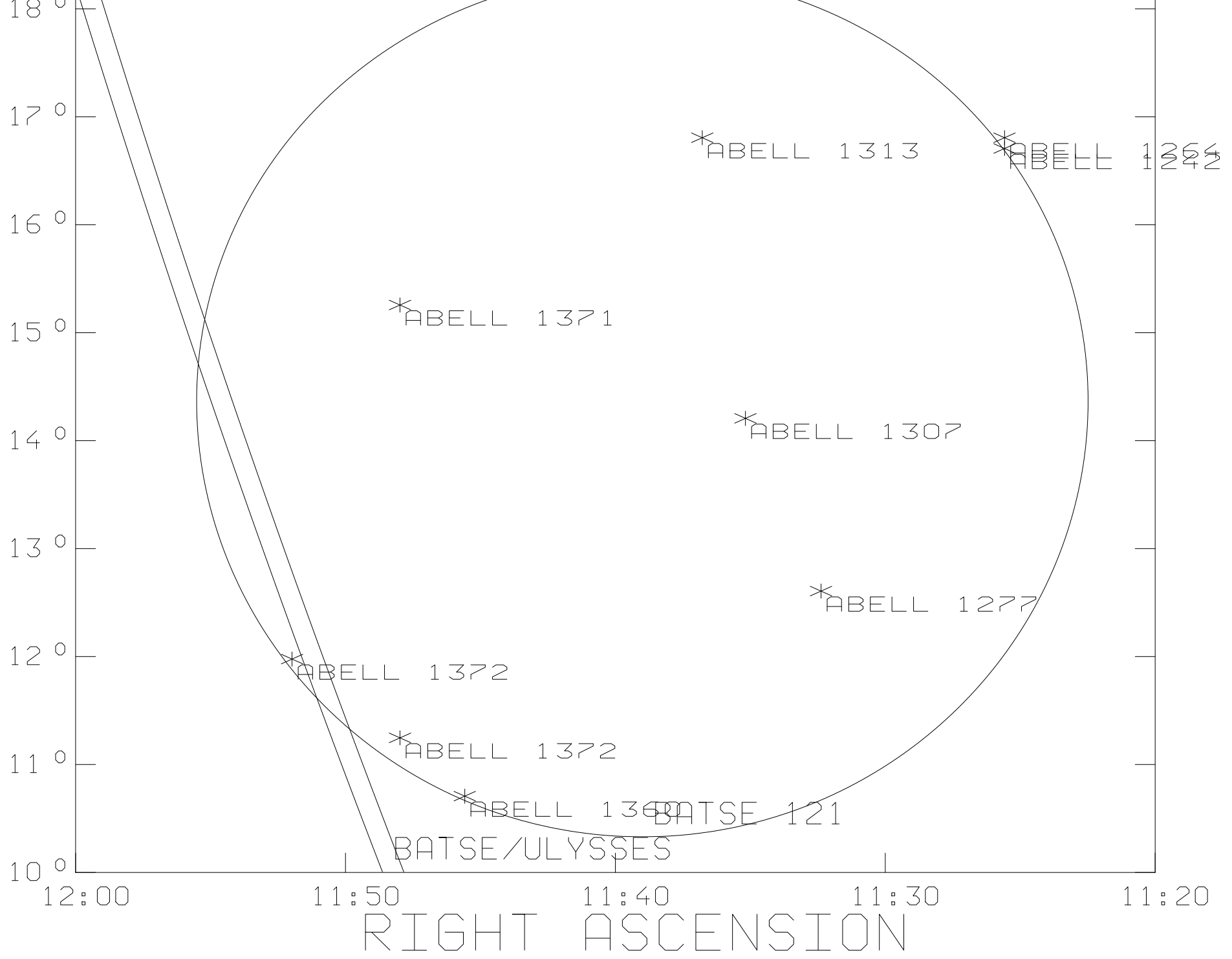

